\title{
Cognitive and Affective Mechanisms of Pain and Fatigue in Multiple Sclerosis
}

\author{
Anne Arewasikporn ${ }^{1,2}$, Aaron P. Turner ${ }^{1,2}$, Kevin N. Alschuler ${ }^{1,3}$, Abbey J. Hughes $^{4}$, and \\ Dawn M. Ehde ${ }^{1}$ \\ ${ }^{1}$ Department of Rehabilitation Medicine, University of Washington School of Medicine \\ ${ }^{2}$ Multiple Sclerosis Center of Excellence-West, Veteran Affairs Puget Sound Health Care System \\ ${ }^{3}$ Department of Neurology, University of Washington School of Medicine \\ ${ }^{4}$ Department of Physical Medicine and Rehabilitation, Johns Hopkins University School of \\ Medicine
}

\begin{abstract}
Objective-To examine the extent to which pain catastrophizing, fatigue catastrophizing, positive affect, and negative affect simultaneously mediated the associations between common symptoms of multiple sclerosis (MS; i.e., pain, fatigue) and impact on daily life, depressive symptoms, and resilience.
\end{abstract}

Methods-Participants were community-dwelling adults with MS $(N=163)$ reporting chronic pain, fatigue, and/or moderate depressive symptoms. Multiple mediation path analysis was used to model potential mediators of pain and fatigue separately, using baseline data from a randomized controlled trial comparing two symptom self-management interventions.

Results-In the pain model, pain catastrophizing was a mediator of pain intensity with pain interference and depression. Negative affect was a mediator of pain intensity with depression and resilience. In the fatigue model, fatigue catastrophizing was a mediator of fatigue intensity with fatigue impact and depression. Positive affect was a mediator of fatigue intensity with depression and resilience.

Conclusions-These findings provide preliminary support for the presence of differential effects of cognitive-affective mediators and suggest potential targets for psychological interventions based on an individual's clinical presentation. The differential mediating effects also support the inclusion of both positive and negative aspects of psychological health in models of pain and fatigue, which would not be otherwise apparent if negative constructs were examined in isolation. To our knowledge, this is the first study to utilize a multivariate path analysis approach to examine cognitive-affective mediators of pain and fatigue in MS, while also examining positive and negative constructs concurrently.

Correspondence concerning this manuscript should be addressed to Anne Arewasikporn, PhD, Department of Rehabilitation Medicine, 325 Ninth Avenue, Box 359612, University of Washington School of Medicine, Seattle, WA 98104. aarewasi@uw.edu. 


\section{Keywords}

catastrophization; affect; pain; fatigue

Chronic pain and fatigue are common symptoms affecting approximately $40-50 \%$ and $90 \%$, respectively, of adults with multiple sclerosis (MS; Finlayson, Johansson, \& Kos, 2012; O'Connor, Schwid, Hermann, Markman, \& Dworkin, 2008). Both are thought to interfere with physical and psychosocial well-being in this population. High levels of pain and fatigue are associated with greater impact on daily life and depressive symptomatology (Bol et al., 2010; Day et al., 2016). These symptoms are also correlated with poorer health, sleep, and quality of life (O'Connor et al., 2008; Motl \& McAuley, 2010), and there is burgeoning evidence that they are also related to low levels of resilience-here defined as the capacity to respond adaptively to stressful situations (Terrell et al., 2014). Given the potential impact that MS-related pain and fatigue intensity may have on positive outcomes like resilience, and negative outcomes such as negative impact on life and depression, researchers have begun to examine potential mechanisms that may explain these associations. Better understanding of mechanisms may ultimately inform psychosocial treatment of pain and fatigue. Four key mediators emerge when considering the relationships among pain intensity, fatigue intensity, disability, depression, and resilience: pain catastrophizing (PC), fatigue catastrophizing (FC), negative affect (NA), and positive affect (PA).

PC and FC refer to disproportionately negative and unrealistic cognitions about pain- and fatigue-related symptoms, respectively (Sullivan, Stanish, Waite, Sullivan, \& Tripp, 1998). The current study treated PC and FC as separate constructs to be consistent with how they have been examined in the research literature. PC has been associated with greater pain interference and distress in samples with MS (Osborne, Jensen, Ehde, Hanley, \& Kraft, 2007). Individuals with MS who tend to engage in high levels of PC and/or FC report more pain, fatigue, psychological distress, and disability (Knoop, van Kessel, \& Moss-Morris, 2012; Lukkahatai \& Saligan, 2013; van Kessel \& Moss-Morris, 2006). Furthermore, longitudinal and intervention studies in non-MS populations with chronic pain have shown changes in PC mediate the reduction of pain intensity (Smeets, Vlaeyen, Kester, \& Knottnerus, 2006; Turner, Holtzman, \& Mancl, 2007), pain interference (Smeets et al., 2006), and depression (Sturgeon \& Zautra, 2013). PC and FC have been associated with poorer outcomes, however their potential mechanistic role in pain and fatigue, respectively, particularly with respect to resilience, has not been well established in samples of people with MS.

NA refers to aversive mood states (e.g., fear, sadness). Like PC and FC, NA is also associated with higher pain and fatigue intensity in MS (Black \& Dorstyn, 2015; Hirsh, Turner, Ehde, \& Haselkorn, 2009). NA has been suggested as a mechanism for pain interference (Hirsh et al., 2009) and depressive symptoms (Kratz, Ehde, \& Bombardier, 2014). In the context of fatigue, low levels of NA have been associated with greater resilience (Black \& Dorstyn, 2015). These studies highlight NA as a potential mediator for pain and fatigue in MS. 
Though related to NA, PA is an independent affective construct that may uniquely influence physical and mental health outcomes (Pressman \& Cohen, 2005; Davis, Zautra, \& Smith, 2004). Evidence of the unique contributions of NA and PA has been found in people with chronic pain (Davis et al., 2004; Sturgeon, Zautra, \& Arewasikporn, 2014) and in an acquired disability sample that included individuals with MS (Alschuler, Kratz, \& Ehde, 2016). Positive emotion is associated with less depressive symptoms (Hart, Vela, \& Mohr, 2008), and induced PA has been shown to reduce pain sensitivity in experimental studies (Finan \& Garland, 2015). PA is also robustly associated with greater resilience (Black \& Dorstyn, 2015; Quale \& Schanke, 2010), and has been implicated in mediating key relationships with pain interference in other rehabilitation samples (e.g., limb loss; Walsh et al., 2016). The relationship between PA and fatigue in MS has not been established. While there is converging evidence that greater fatigue is associated with low levels of PA (e.g., chronic pain: Zautra, Fasman, Parish, \& Davis, 2007; chronic fatigue syndrome: Marshall et al., 1996), findings in the MS literature are mixed (Krupp et al., 2002; Black \& Dorstyn, 2015). Although PC, FC, NA, and PA have been suggested as mechanisms of pain and fatigue in non-MS populations, their role as mechanisms in MS populations is less clear.

\section{The Present Study}

The purpose of the present study was to examine whether PC, FC, NA, and PA mediated the cross-sectional relationships between common symptoms of MS (pain intensity, fatigue intensity) and important criterion measures (pain interference, fatigue impact, depressive symptoms, resilience) in separate models for pain and fatigue. Specifically, we expected the following direct effects: pain intensity and fatigue intensity would be associated with greater PC and FC respectively, higher NA, and lower PA; and that each of these potential mediating variables would be associated with greater negative impact on daily life, more depressive symptomatology, and less resilience. With respect to indirect effects, we hypothesized that PC (for pain) and FC (for fatigue), NA, and PA would each mediate each symptom-criterion relationship. We also anticipated that NA and PA would be intermediaries in the pain intensity-PC and fatigue intensity-FC relationships, as we proposed pain- and fatigue-related emotional responses could be followed by negative cognitive appraisals. Significant indirect effects were interpreted as preliminary support for the mediating variables' potentially central role in the associations described. This study was the first of any design to explore these relationships together within a model.

\section{Method}

\section{Design}

This study was a secondary analysis of baseline data from a randomized clinical trial comparing two self-management interventions for depression, fatigue, and/or pain in adults with MS (see Ehde et al., 2015). The original study did not examine relationships among the present study's variables of interest. The University of Washington institutional review board approved all study procedures. Data were collected between April 2011 and September 2013. 


\section{Sample and Recruitment}

Participants were originally recruited for the randomized clinical trial via mailings through the University of Washington Department Research Registry, advertisement through national MS organizations, flyers and referrals from the University of Washington MS Center, the ClinicalTrials.gov website, and through other active studies within the department. Eligible individuals were 18 years of age or older with a self-reported diagnosis of MS who demonstrated one or more of the following criteria: (1) moderate depressive symptoms indicated with Patient Health Questionnaire-9 (PHQ-9) with scores ranging from 10 to 14 (Kroenke, Spitzer, \& Williams, 2001; (2) chronic pain defined as an average pain intensity of 3 or higher on the 0-10 Numeric Pain Rating Scale (Jensen \& Karoly, 1992); or (3) significant fatigue symptoms defined as a score of 10 or higher on the Modified Fatigue Impact Scale (MFIS) Short Form (Fisk et al., 1994). Exclusionary criteria included severe cognitive impairment ( $\geq 1$ error on the six-item Cognitive Screener; Callahan, Unverzagt, Hui, Perkins, \& Hendrie, 2002); current participation in psychotherapy; participation in another study for fatigue, depression, or pain in MS; and moderate-severe to severe depressive symptoms (i.e., PHQ-9 score > 15). Additional details concerning study design can be found in Ehde et al., 2015.

\section{Measures}

Demographic and disease-related information-Age, sex, race, ethnicity, marital status, years of education, and disease duration were self-reported during the initial baseline assessment. MS subtype was assessed with a validated self-report item that utilized written description and visual depictions of the major MS disease subtypes (Bamer, Cetin, Amtmann, Bowen, \& Johnson, 2007). Level of disability was assessed with the PatientDetermined Disease Steps Scale (Learmonth, Motl, Sandroff, Pula, \& Cadavid, 2013).

Pain intensity-The Numeric Rating Scale is a self-report measure that has been used to assess pain severity and has been validated for use for individuals with MS (Jensen \& Karoly, 1992; Dworkin et al., 2005). Participants were asked to rate the intensity of their current, least, worst, and average pain in the past week, on a 0 (no pain) to 10 (pain as bad as you can imagine) scale. A composite score was created by taking the mean of these four ratings. Internal consistency was good (Cronbach's $a=.88$ ).

Fatigue intensity-A single item from the Multidimensional Assessment of Fatigue Scale was used to assess fatigue severity (Tack, 1990). Participants were asked, "To what degree have you experienced fatigue in the past week?" on a 0 (not at all) to 10 (a great deal) scale.

Pain catastrophizing-The Pain Catastrophizing Scale (PCS) was used to assess PC (Sullivan, 1995). The PCS is comprised of 13 pain-related statements such as, "I worry all the time about whether the pain will end." Participants were asked to rate the degree to which they had these negative cognitions while experiencing pain on a 0 (not at all) to 4 (all the time) scale. The internal consistency of this scale was excellent $(\alpha=.94)$.

Fatigue catastrophizing-The PCS was modified to assess FC by substituting "fatigue" for "pain." This adapted scale has been used to examine FC in individuals with MS (Bol et 
al., 2010) and chronic fatigue syndrome (Romano et al., 2016). Participants were asked to rate the extent to which they experienced 13 negative cognitions while experiencing fatigue on a 0 (not at all) to 4 (all the time) scale. The internal consistency of this scale was excellent $(a=.94)$, with "if item deleted" alphas ranging from .93 to .94 . Because this scale has - to our knowledge — not been validated in individuals with MS, we evaluated the psychometric properties of the measure with confirmatory factor analysis. Fit statistics for the initial model indicated borderline model fit $\left[\mathrm{X}^{2}(65)=152.95, p<.001\right.$; RMSEA = .09; $\mathrm{CFI}=.91 ; \mathrm{SRMR}=.05]$ based on Hu and Bentler's (1999) benchmarks for good fit (CFI >. 95; RMSEA<.06; SRMR<.08). The modification indices suggested several areas of overlap among the FC items. Correlating the residuals of item pairs with overlapping content improved model fit to acceptable levels, $\left[\mathrm{X}^{2}(60)=91.05, p=.006 ; \mathrm{RMSEA}=.06 ; \mathrm{CFI}=.97\right.$; SRMR $=.04]$. All standardized factor loadings were significant. The analysis supported an acceptable one-factor solution.

Positive affect-The Positive and Negative Affect Scale (PANAS; Watson, Clark, \& Tellegen, 1988) was used to measure PA. A list of 10 positive emotion adjectives was presented to the participant (e.g., "interested," "excited"). Participants were asked to rate the extent to which they experienced these emotions during the past week, from 1 (very slightly/not at all) to 5 (extremely). The internal consistency of this scale was good ( $a=$ 89).

Negative affect-The PANAS was also used to measure NA (Watson et al., 1988). Using the same rating scale and timeframe, participants rated how much they had experienced 10 negative emotions (e.g., "distressed," "guilty,") on a 1 (very slightly/not at all) to 5 (extremely) scale. The internal consistency of this scale was also good $(a=.86)$.

Pain interference-The seven-item Brief Pain Inventory (BPI) Interference Scale was adapted for use in MS (Tan, Jensen, Thornby, \& Shanti, 2004). Participants were asked to rate pain interference on a scale of 0 (does not interfere) to 10 (completely interferes) for general activity, mood, mobility, work, relations with other people, sleep, and enjoyment in life. Internal consistency for this scale was excellent $(a=.92)$.

Fatigue impact-The MFIS contains 21 items used to assess the perceived impact of fatigue in physical, cognitive, and psychosocial domains (Fisk et al., 1994). Participants were asked to rate the frequency with which fatigue influenced a variety of activities (e.g., "I have been unable to think clearly") on a scale ranging from 0 (never) to 4 (almost always). The MFIS has been validated for use in MS (Rietberg, van Wegan, \& Kwakkel, 2010; Téllez et al., 2005). Internal consistency for this scale was excellent $(a=.92)$.

Depressive symptoms-Depression was evaluated with the nine-item PHQ-9 (Kroenke et al., 2001). Participants were asked to report the frequency of depressive symptoms in the last two weeks on a 0 (not at all) to 3 (nearly every day) scale. Validity of the PHQ-9 has been established for MS populations (Sjonnesen et al., 2012; Williams et al., 2005).

Resilience-The 10-item Connor Davison Resilience Scale was used to assess resilience (Connor \& Davidson, 2003). Participants were asked to rate the extent to which they 
demonstrated different aspects of resilience (e.g., "able to adapt to change," "coping with stress strengthens me," on a 5-point scale from 0 (not true at all) to 4 (true nearly all the time). The internal consistency of this scale was good $(a=.88)$.

\section{Data Analytic Plan}

SPSS 23 (IBM Corp., 2015) was used to obtain descriptive statistics and examine statistical assumptions (normality, skewness, kurtosis) of the study variables. To test the study hypotheses, we constructed separate models for pain and fatigue and utilized multiple mediation path analysis (i.e., a form of structural equation modeling that only uses observed variables) with the TYPE = GENERAL command in Mplus 7.6 (Muthén \& Muthén, 1998). We selected this approach for several reasons. It allowed us to estimate the strength of an association between two variables while accounting for shared variance among the study variables in the model, resulting in a "purer" estimate of the association. The use of multiple mediation path analysis also allowed us to estimate all hypothesized indirect effects simultaneously and detect potential separate pathways between variables. The Root Mean Square Error of Approximation (RMSEA), Bentler's Comparative Fit Index (CFI), and the Standardized Root Mean Square Residual (SRMR) were used to assess model fit, with adequate fit meeting the following criteria: RMSEA $<.08$, CFI $>.95$, SRMR $<.08$. Potential covariates (i.e., demographic and disease-related characteristics) were then entered individually into the models. Significant covariates [(e.g., sex, age, race, employment status, level of disability, and MS type (dichotomized into relapse-remitting vs. all other progressive subtypes)] were retained in the final models. Indirect effects were calculated using the $a b$ product of the coefficients; where the a path refers to the relationship between the independent variable and the mediating variable, and the $b$ path refers to the relationship between the mediating variable and the criterion variable. The pain model examined PC, NA, and PA as mediators of the relationship between pain intensity and each criterion variable (pain interference, depression, resilience). For instance, we hypothesized that PC would be a mediator of pain intensity with pain interference, depression, and resilience. We also predicted that NA would mediate the same associations, as would PA. NA and PA were also examined as mediators of the relationship between pain intensity and PC. Thus we expected to find a total of 11 mediating effects in the pain model. Similar to the pain model, the fatigue model examined FC, NA, and PA as mediators of fatigue intensity with fatigue impact on life, depression, and resilience; NA and PA were also examined as mediators of the relationship between fatigue intensity and FC, for a total of 11 indirect effects. R-squared values were computed to evaluate effect sizes, with $r=.10, .30$, and .50 representing small, medium, and large effects, respectively (Cohen, 1988). Although our analyses did not fully support the inferences of mediation (i.e., temporal precedence) due to the cross-sectional nature of the data, it would be more appropriate to refer to the mediators as "intervening variables" (Mackinnon, Lockwood, Hoffman, \& West, 2002). With this in mind, we employed the term "mediation" to refer to the analytic approach of estimating the degree to which intervening variables accounted for the relationship between the independent variable and criterion. Because cross-sectional mediation can result in overestimation of true direct and indirect effects (Sheikh, Abelsen, \& Olsen, 2016), significant results were interpreted as preliminary findings. 


\section{Results}

\section{Participants}

The sample was comprised of individuals with MS living across the United States (see Table 1). Participants had a mean age of 52.1 years ( $S D=10.1$, range: $25-76)$, were predominantly female (87.1\%), non-Hispanic white (83.4\%), married (61.3\%), and highly educated (median=college degree or higher). Approximately 55.8\% of participants reported having relapse-remitting MS, 20.9\% secondary-progressive, $17.2 \%$ primary-progressive, and $4.9 \%$ progressive-relapsing. Mean disease duration was 12.0 years ( $\mathrm{SD}=9.0$, range: 0-49). Level of disability ranged widely from no impairment to requiring a wheelchair or scooter (median=gait impairment without need for an assistive device).

\section{Pain Model}

The pain model demonstrated adequate fit, $\chi^{2}(31)=19.66, p=.018, \mathrm{RMSEA}=.061$, CFI $=.965, \mathrm{SRMR}=.060$. Medium effect sizes were found for PA and NA $\left(r_{\mathrm{PA}}=0.29 ; r_{\mathrm{NA}}=\right.$ $0.36)$; and large effect sizes were found for PC $(r=0.65)$, pain interference $(r=0.85)$, depression $(r=0.59)$, and resilience $(r=0.53$; see Table $\mathrm{S} 1$ for additional information).

Direct effects-Consistent with our hypotheses, pain intensity was associated with greater PC, NA, and pain interference, however it was not related to PA (see Figure 1 for standardized parameter estimates). PC was associated with more pain interference and depression as expected, but it was not related to resilience. NA was associated with more depression, less resilience, and marginally more pain interference. PA was associated with less depression and more resilience, but it was not related to pain interference. More NA and less PA were associated with greater PC, as hypothesized.

Indirect effects-PC significantly mediated the relationship between pain intensity and pain interference $(a b=0.19, p<.001)$. It also mediated the relationship between pain intensity and depression $(a b=0.11, p=.007)$. NA mediated the relationships between pain intensity and depression $(a b=0.07, p=.04)$, and pain intensity and resilience ( $a b=-0.07, p$ $=.04$ ). A marginal effect for NA was also found, such that it mediated the relationship between pain intensity and PC $(a b=0.05, p=.09)$. PA did not mediate any relationships between pain intensity and the criterion variables $\left(a b_{\mathrm{BPI}}=0.0001, p=.88 ; a b_{\mathrm{PHQ} 9}=0.003\right.$, $\left.p=.88 ; a b_{\text {RESILIENCE }}=-0.007, p=.88 ; a b_{\mathrm{PC}}=0.002, p=.88\right)$.

\section{Fatigue Model}

The fatigue model demonstrated fair fit, $\chi^{2}(27)=59.97, p=.0003$, RMSEA $=.087$, CFI $=$. 925 , SRMR $=.066$. Like the pain model, Pearson $r$ values suggested medium effect sizes for PA and NA ( $\left.r_{\mathrm{PA}}=0.33 ; r_{\mathrm{NA}}=0.32\right)$, and large effect sizes for FC $(r=0.59)$, fatigue impact $(r=0.63)$, depression $(r=0.62)$, and resilience $(r=0.73$; see Table $\mathrm{S} 1$ for additional information).

Direct effects-In support of our hypotheses, fatigue intensity was associated with greater FC, less PA, and more fatigue impact (Figure 2). Fatigue intensity was not related to NA. FC was associated with more fatigue impact and depression, but it was not related to resilience. 
As expected, NA was associated with more depression and less resilience, but it was not associated with fatigue impact. PA was associated with less depression, more resilience, and marginally less fatigue impact. NA — but not PA—was associated with more FC.

Indirect effects-FC significantly mediated the relationship between fatigue intensity and fatigue impact $(a b=0.12, p=.001)$. It also mediated the relationship between fatigue intensity and depression $(a b=0.08, p=.01)$. PA mediated the relationship between fatigue intensity and depression $(a b=0.05, p=.04)$, as well as the relationship between fatigue intensity and resilience $(a b=-0.12, p=.006)$. NA did not mediate the relationships between fatigue intensity and the criterion variables $\left(a b_{\mathrm{MFIS}}=0.001, p=.89 ; a b_{\mathrm{PHQ}}=-0.004, p=\right.$. $\left.88 ; a b_{\text {RESILIENCE }}=0.005, p=.88\right)$. PA and NA did not mediate the relationship between fatigue intensity and FC $\left(a b_{\mathrm{PA}}=0.02, p=.20 ; a b_{\mathrm{NA}}=-0.006, p=.88\right)$.

\section{Discussion}

The results of the present study demonstrated that PC, FC, NA, and PA differentially mediated the associations of symptoms (pain and fatigue) with pain interference, fatigue impact, depression, and resilience. PC and NA emerged as significant mediators in the pain model, whereas FC and PA were significant mediators in the fatigue model. We further examined the relationship between pain intensity and PC, and fatigue intensity and FC, in the pain and fatigue models, respectively; however NA and PA did not significantly mediate the relationships. The differential mediating effects support further study of the possible mechanistic role that PC, FC, NA, and PA play in the context of pain and fatigue in MS; and may have implications for further development of psychosocial interventions. To our knowledge, this study was the first to explore these relationships together.

\section{Potential Cognitive Mechanisms}

Our hypotheses involving PC and FC were partially supported. For both pain and fatigue, PC and FC appear to account for the relationship between symptoms and impact on daily life and depression. Our findings suggest that the degree to which pain intensity and fatigue intensity are associated with negative mood and other aspects of daily life may be determined in part by how one thinks about these symptoms. The mediating effect of PC in these associations is consistent with past research in chronic pain samples (Osborne et al. 2007; Smeets et al., 2006) and extends findings to MS samples. FC appeared to function similarly in the fatigue model, also suggesting that FC may be potentially important in the analogous associations in the fatigue model. PC and FC were not associated with resilience, as initially theorized, a finding that was inconsistent with research that suggests that more resilient individuals with chronic pain catastrophize about pain less than individuals reporting low levels of resilience (Ong, Zautra, \& Reid, 2010). Further examination of PC and FC and their associations with pain intensity and fatigue intensity showed our results were inconsistent with the hypothesis that PA and NA served as intermediaries in those relationships, though it is possible that these relationships may be difficult to observe over long periods of time, and may only be apparent when examined at a momentary level of analysis. Additional studies that can establish the temporal order of these variables are needed to more thoroughly evaluate if this is the case. 
Consistent with well-established theory proposed by Turk (1978), the present findings provide preliminary support that PC may serve a mechanistic role in the pain intensity with pain interference and depression associations, and that FC appeared to function similarly in the fatigue model, consistent with van Kessel's (2006) more recent cognitive-behavioral model of fatigue. However, these possible relationships need to be confirmed with longitudinal study designs, which permit tests of causality. Conceptually, the findings support further use of a cognitive-behavioral model (Bol et al., 2010; Romano et al., 2016) in MS, and are consistent with research demonstrating the efficacy of cognitive-behavioral therapy for fatigue (e.g., van den Akker et al., 2016; Mohr, Hart, \& Goldberg, 2003). Targeting FC with cognitive restructuring in the same way PC is typically targeted for pain reduction in cognitive-behavioral therapy for pain could be a useful strategy for reducing fatigue impact and dysphoric mood. The similar pattern of direct and indirect effects raise questions about the extent to which PC and FC tap into a more global concept representing general catastrophizing. It is plausible that individuals who catastrophize about pain would also be more likely to catastrophize about fatigue, and indeed we found a positive correlation between the two measures $(r=.63, p<.001)$. Future studies ought to examine whether symptom-specific catastrophizing is indicative of an underlying characteristic of the individual, as opposed to a coping strategy that is symptom-specific. Though PC and FC were not related to resilience in our models, other, same-valence cognitive styles such as benefit finding or positive reappraisal (Yeung, Arewasikporn, \& Zautra, 2012) may account for the relationship between symptoms and resilience, and ought to be examined in future research.

It is important to note that reasonable arguments are available for the reverse sequence of effects. Though we hypothesized a specific sequence among the variables (e.g., greater fatigue intensity is associated with increased FC, which increases fatigue impact and depression), it is possible that having greater depression and fatigue-related disruption in daily life may also lead to more severe fatigue through increases in fatigue catastrophizing. Indeed, other studies of chronic pain have suggested that the relationship between pain intensity and PC may be bidirectional (Janssen, 2002). Regardless of whether the sequence was as hypothesized, reversed, or both, the central role of PC and FC as mediators remains unchanged.

\section{Potential Affective Mechanisms}

In the present study, affect appeared to have an important role in the relationships involving depression and resilience, though in different ways for pain and fatigue. Some of our results were consistent with past research, while other results differed. In the pain model, the pain intensity-depression and pain intensity-resilience associations occurred through NA. The primary role of NA in these relationships is consistent with research that shows negative emotion associated with pain facilitates the development of depression in chronic pain (Brown, 1990). In contrast, PA did not mediate the relationship between pain intensity and resilience (nor any other criterion variable), a surprising result given the robust findings in the literature that suggest resilient functioning occurs via PA (e.g., Quale \& Schanke, 2010). In the fatigue model, PA mediated the fatigue intensity-depression and fatigue intensityresilience relationships, consistent with our hypotheses and research in chronic pain (Zautra, 
et al., 2007) and chronic fatigue syndrome (Marshall et al., 1996). However contrary to our hypotheses, NA did not mediate the same relationships.

In line with prior research on the emotional consequences of pain (see Gatchel, Peng, Peters, Fuch, \& Turk, 2007 for a review), the pain model findings support further study of NA as a potential mechanism for pain, to see if having aversive experiences, rather than the absence of positive ones, may be what perpetuates the relationships among pain intensity, depression, and resilience in MS. Though, it is possible that we did not find significant mediation effects for PA because it may be more likely to modify the relationships between pain intensity and the criterion variables, attenuating the relationships (Finan \& Garland, 2015). A different rendering of the relationships among symptoms, affect, and criterion variables emerged for the fatigue model, providing additional clarity about the role of affect in MS fatigue. To our knowledge, this is the first study to suggest PA—but not NA-may serve as an important mechanism for fatigue. Thus, there may be some benefit to focusing on positive emotion when considering the relationship between fatigue intensity, depression, and resilience. Interventions that increase PA may be effective in mitigating depressive symptoms and encouraging resilience. Alternately, it is possible that our negative findings for NA were related to methodological differences in measurement. In the present study, state NA (i.e., in the past week) was assessed while other studies in the literature assessed for trait NA (de Ridder et al., 2004). We also controlled for PA in our analyses, while these other studies did not. These or other unidentified differences may have contributed to the incongruity. Given the small body of literature on fatigue and affect, additional studies are needed to further elucidate the potential mediating role of PA and NA in the relationships among pain intensity, fatigue intensity, depression, and resilience.

While the previously described model has been supported in the literature, we acknowledge that the effects could have occurred in reverse sequence. For instance, it is possible that individuals who have greater depressive symptom severity may evidence greater NA and consequently, report greater pain intensity. Findings to this effect have been found in chronic pain samples (Gatchel et al., 2007), however as we stated above, the contributions of NA and PA as mediators for pain and fatigue respectively do not depend on the direction of the effects.

In sum, there were many similarities between the pain and fatigue models-namely with respect to PC and FC and their relationship with impact on daily life and depressionhowever, the differences between the models represent a novel finding: NA partially accounts for the associations among pain intensity, depression, and resilience; whereas PA partially accounts for the associations among fatigue intensity, depression, and resilience. These findings have potentially important implications for psychosocial interventions, particularly with regard to therapeutic targets. Patient factors, including baseline levels of cognitive and affective variables, should be considered in tailoring the intervention strategies and targets. Assuming causality has been confirmed in the hypothesized directions, reducing fatigue impact and depression could potentially be achieved in an individual reporting severe fatigue through cognitive restructuring to decrease FC and pleasant activities scheduling to increase PA. Alternately, if the reverse order of effects is true and one wishes to reduce fatigue intensity; reducing fatigue impact, managing depression, and bolstering resilience via 
the same mediators could be beneficial. These findings also suggest that intervention strategies that increase PA (e.g., behavioral activation, social engagement, mindful selfcompassion, gratitude exercises, physical activity based on personally meaningful goals) may be important treatment components in psychosocial interventions for fatigue among individuals with MS. Developing and testing psychosocial interventions based on these models represent important next steps in the management of pain, fatigue, and mood in MS.

In contrast to the approach taken in prior studies of MS, our analytic strategy-multiple mediator path analysis-allowed us to more accurately reflect the complex relationships that exist between commonly experienced symptoms of MS, and represents a strength of the study. Numerous studies have examined the associations between common symptoms of MS, however, few studies in the MS literature have utilized multiple mediator path analysis to examine concurrent relationships among predictors, mediators, and outcome variables. Of the few, only one study included positively-oriented constructs (e.g., PA, resilience) and none statistically tested proposed mechanisms with mediation analyses (Black \& Dorstyn, 2015; Bol et al., 2010; Day et al., 2016). This statistical approach allowed us to evaluate the mediating effects of PC, FC, PA, and NA concurrently while controlling for the statistical overlap among constructs. To our knowledge, this is the first published study to use this data analytic tool to examine cognitive and affective mediators of pain and fatigue in MS, while also including both positive and negative aspects of mood and psychological health.

\section{Limitations}

Several limitations of the current study should be acknowledged. First, the sample was, by design, composed of adults who reported chronic pain, fatigue, and/or moderate depression. Thus, the findings may not generalize to all adults with MS, particularly to those who experience less frequent and milder levels of pain and fatigue, severe depression, and severe cognitive impairment. Second, the study utilizes cross-sectional data, which while informative, cannot definitively establish causality. It is also possible that reverse causal effects or bidirectional associations exist between variables, as previously discussed. Furthermore, the estimates of the direct and indirect effects may be overestimated, as there is some evidence of increased Type I error rates in cross-sectional mediation (Sheikh et al., 2016). Finally, the study did not include sleep, other MS symptoms, and medication use known to potentially influence the study variables. Despite these limitations, it is still possible to derive meaningful information about potential cognitive and affective mechanisms underlying the relationships among pain intensity, fatigue intensity, impact on daily life, depression, and resilience. Taken together, they present a theoretical model that can be tested in future studies designed to evaluate causal relationships.

\section{Future Directions}

Future studies would benefit from using methodology that can establish causality between the variables examined in the present study. Manipulation of pain, fatigue, catastrophizing, PA, and/or NA in laboratory paradigms, and examination of lagged effects in time series or longitudinal data would provide stronger support for causality. Future studies should also examine associations among the examined variables at the within-person or state-level. The present study evaluated relationships between the examined variables at the between-person 
level, examining how people who tend to experience more fatigue, for example, report less PA and more depression. The use of ecological momentary assessment or daily diaries allows one to examine how momentary or day-to-day changes in state-level pain and fatigue influence mediators and outcomes at the state-level. For instance, in momentary assessments one could examine the influence of morning pain on end-of-day mood within-person, and test whether midday PA mediates the morning pain-evening mood relationship. This approach has the added benefit of enhanced ecological validity and the ability to establish temporal precedence.

\section{Conclusions}

This study is the first to examine cognitive and affective mediators of pain intensity, fatigue intensity, pain interference, fatigue impact, depression, and resilience in a sample of adults with MS. Our results suggest higher levels of pain interference, fatigue impact, and depression were associated with for more intense pain and fatigue through greater PC and FC respectively. Lower levels of depression and greater resilience were associated with less severe pain and fatigue through NA and PA, respectively. This study highlights the need for comprehensive modeling of symptoms in longitudinal or experimental designs, and suggests future interventions may potentially benefit from differentially targeting these constructs.

\section{Supplementary Material}

Refer to Web version on PubMed Central for supplementary material.

\section{Acknowledgments}

The contents of this manuscript were developed under grants from National Institute on Disability, Independent Living, and Rehabilitation Research (NIDILRR grant number H133B080025, Dawn Ehde, Project P.I.) and the National Multiple Sclerosis Society (MB0026, Aaron Turner, P.I.). NIDILRR is a Center within the Administration for Community Living (ACL), Department of Health and Human Services (HHS). The contents of this manuscript do not necessarily represent the policy of NIDILRR, ACL, HHS, and you should not assume endorsement by the Federal Government. Some of this study's findings were presented at the 18th Annual Rehabilitation Psychology Conference on February 20, 2016, in Atlanta, Georgia.

\section{References}

Alschuler KN, Kratz AL, Ehde DM. Resilience and vulnerability in individuals with chronic pain and physical disability. Rehabilitation Psychology. 2016; 61(1):7-18. DOI: 10.1037/rep0000055 [PubMed: 26881303]

Bamer AM, Cetin K, Amtmann D, Bowen JD, Johnson KL. Comparing a self report questionnaire with physician assessment for determining multiple sclerosis clinical disease course: A validation study. Multiple Sclerosis. 2007; 13(8):1033-1037. DOI: 10.1177/1352458507077624 [PubMed: 17468438]

Black R, Dorstyn D. A biopsychosocial model of resilience for multiple sclerosis. Journal of Health Psychology. 2015; 20(11):1434-1444. DOI: 10.1177/1359105313512879 [PubMed: 24323335]

Bol Y, Duits AA, Lousberg R, Hupperts RM, Lacroix MH, Verhey FR, Vlaeyen JW. Fatigue and physical disability in patients with multiple sclerosis: A structural equation modeling approach. Journal of Behavioral Medicine. 2010; 33(5):355-363. DOI: 10.1007/s10865-010-9266-8 [PubMed: 20508981]

Brown GK. A causal analysis of chronic pain and depression. Journal of Abnormal Psychology. 1990; 99(2):127-137. [PubMed: 2348006] 
Callahan CM, Unverzagt FW, Hui SL, Perkins AJ, Hendrie HC. Six-item screener to identify cognitive impairment among potential subjects for clinical research. Medical Care. 2002; 40(9):771-781. DOI: 10.1097/01.MLR.0000024610.33213.C8 [PubMed: 12218768]

Cohen, J. Statistical power analysis for the behavioral sciences. 2nd. New York, NY: Lawrence Erlbaum Associates; 1988.

Connor KM, Davidson JRT. Development of a new resilience scale: The Connor-Davidson Resilience Scale (CD-RISC). Depression and Anxiety. 2003; 18:76-82. DOI: 10.1002/da.10113 [PubMed: 12964174]

Davis MC, Zautra AJ, Smith BW. Chronic pain, stress, and the dynamics of affective differentiation. Journal of Personality. 2004; 72(6):1133-1160. DOI: 10.1111/j.1467-6494.2004.00293.x [PubMed: 15509279]

Day MA, Ehde DM, Ward LC, Hartoonian N, Alschuler KN, Turner AP, Jensen MP. An empirical investigation of a biopsychosocial model of pain in multiple sclerosis. Clinical Journal of Pain. 2016; 32(2):155-163. DOI: 10.1097/AJP.0000000000000240 [PubMed: 26741742]

de Ridder D, Fournier M, Bensing J. Does optimism affect symptom report in chronic disease? Journal of Psychosomatic Research. 2004; 56:341-350. DOI: 10.1016/S0022-3999(03)00034-5 [PubMed: 15046972]

Dworkin RH, Turk DC, Farrar JT, Haythornthwaite JA, Jensen MP, Katz NP, Witter J. Core outcome measures for chronic pain clinical trials: IMMPACT recommendations. Pain. 2005; 113(1-2):919. DOI: 10.1016/j.pain.2004.09.012 [PubMed: 15621359]

Ehde DM, Elzea JL, Verrall AM, Gibbons LE, Smith AE, Amtmann D. Efficacy of a telephonedelivered self-management intervention for persons with multiple sclerosis: a randomized controlled trial with a one-year follow-up. Archives of Physical Medicine and Rehabilitation. 2015; 96(11):1945-1958. DOI: 10.1016/j.apmr.2015.07.015 [PubMed: 26254948]

Finan PH, Garland EL. The role of positive affect in pain and its treatment. Clinical Journal of Pain. 2015; 31(2):177-187. DOI: 10.1097/AJP.0000000000000092 [PubMed: 24751543]

Finlayson, M., Johansson, S., Kos, D. Fatigue. In: Finlayson, M., editor. Multiple sclerosis rehabilitation: From impairment to participation. Boca Raton, FL: CRC Press; 2012. p. 69-100.

Fisk JD, Ritvo PG, Ross L, Haase DA, Marrie TJ, Schlech WF. Measuring the functional impact of fatigue: Initial validation of the Fatigue Impact Scale. Clinical Infectious Diseases. 1994; 18(Suppl1):S79-S83. DOI: 10.1093/clinids/18.Supplement_1.S79 [PubMed: 8148458]

Gatchel RJ, Peng YB, Peters ML, Fuchs PN, Turk DC. The biopsychosocial approach to chronic pain: Scientific advances and future directions. Psychological Bulletin. 2007; 133(4):581-624. DOI: 10.1037/0033-2909.133.4.581 [PubMed: 17592957]

Hart SL, Vella L, Mohr DC. Relationships among depressive symptoms, benefit-finding, optimism, and positive affect in multiple sclerosis patients after psychotherapy for depression. Health Psychology. 2008; 27(2):230-238. DOI: 10.1037/0278-6133.27.2.230 [PubMed: 18377142]

Hirsh AT, Turner AP, Ehde DM, Haselkorn JK. Prevalence and impact of pain in multiple sclerosis: physical and psychologic contributors. Archives of Physical Medicine and Rehabilitation. 2009; 90(4):646-651. DOI: 10.1016/j.apmr.2008.10.019 [PubMed: 19345781]

$\mathrm{Hu}$ L, Bentler PM. Fit indices in covariance structure modeling: Sensitivity to underparameterized model misspecification. Psychological Methods. 1998; 3(4):424-453. DOI: 10.1037/1082-989X. 3.4.424

Corp, IBM. IBM SPSS Statistics for Mac, Version 23.0. Armock, NY: IBM Corp; 2015.

Janssen SA. Negative affect and sensitization to pain. Scandinavian Journal of Psychology. 2002; 43(2):131-137. DOI: 10.1111/1467-9450.00278 [PubMed: 12004950]

Jensen, MP., Karoly, P. Self-report scales and procedures for assessing pain in adults. In: Turk, DC., Melzack, R., editors. Handbook of Pain Assessment. New York, NY: Guilford Press; 1992. p. 135-151.

Knoop H, Van Kessel K, Moss-Morris R. Which cognitions and behaviours mediate the positive effect of cognitive behavioural therapy on fatigue in patients with multiple sclerosis? Psychological Medicine. 2012; 42(1):205-213. DOI: 10.1017/S0033291711000924 [PubMed: 21672300] 
Kratz AL, Ehde DM, Bombardier CH. Affective mediators of a physical activity intervention for depression in multiple sclerosis. Rehabilitation Psychology. 2014; 59(1):57.doi: 10.1037/a0035287 [PubMed: 24611925]

Kroenke K, Spitzer RL, Williams JBW. The PHQ-9: Validity of a brief depression severity measure. Journal of General Internal Medicine. 2001; 16(9):606-613. DOI: 10.1046/j. 1525-1497.2001.016009606.x [PubMed: 11556941]

Krupp LB, Christodoulou C, Madigan D, Morgan T, Scherl WF, Melville P, McIlree C. The use of inteferon-beta-1a (Avonex) and modafinil to evaluate and treat cytokine-induced fatigue in multiple sclerosis. Annals of Neurology. 2002; 52(3S):S87.

Learmonth YC, Motl RW, Sandroff BM, Pula JH, Cadavid D. Validation of patient determined disease steps (PDDS) scale scores in persons with multiple sclerosis. BMC Neurology. 2013; 13:37.doi: 10.1186/1471-2377-13-37 [PubMed: 23617555]

Lukkahatai N, Saligan LN. Association of catastrophizing and fatigue: A systematic review. Journal of Psychosomatic Research. 2013; 74(2):100-109. DOI: 10.1016/j.jpsychores.2012.11.006 [PubMed: 23332523]

MacKinnon DP, Lockwood CM, Hoffman JM, West SG, Sheets V. A comparison of methods to test mediation and other intervening variable effects. Psychological Methods. 2002; 7(1):83-104. DOI: 10.1037/1082-989X.7.1.83 [PubMed: 11928892]

Marshall PS, Watson D, Steinberg P, Cornblatt B, Peterson PK, Callies A, Schenck CH. An assessment of cognitive function and mood in chronic fatigue syndrome. Biological Psychiatry. 1996; 39(3): 199-206. DOI: 10.1016/0006-3223(95)00131-X [PubMed: 8837981]

Mohr DC, Hart SL, Goldberg A. Effects of treatment for depression on fatigue in multiple sclerosis. Psychosomatic Medicine. 2003; 65(4):542-547. DOI: 10.1097/01.PSY.0000074757.11682.96 [PubMed: 12883103]

Motl RW, McAuley E. Symptom cluster and quality of life: Preliminary evidence in multiple sclerosis. The Journal of Neuroscience Nursing: Journal of the American Association of Neuroscience Nurses. 2010; 42(4):212-216. DOI: 10.1097/JNN.0b013e3181e26c5f [PubMed: 20804116]

Muthén, LK., Muthén, BO. Mplus User's Guide. Seventh. Los Angeles, CA: Muthén \& Muthén; 1998.

O'Connor AB, Schwid SR, Herrmann DN, Markman JD, Dworkin RH. Pain associated with multiple sclerosis: Systematic review and proposed classification. Pain. 2008; 137(1):96-111. DOI: 10.1016/j.pain.2007.08.024 [PubMed: 17928147]

Ong AD, Zautra AJ, Reid MC. Psychological resilience predicts decreases in pain catastrophizing through positive emotions. Psychology and Aging. 2010; 25(3):516-523. DOI: 10.1037/a0019384 [PubMed: 20853962]

Osborne TL, Jensen MP, Ehde DM, Hanley MA, Kraft G. Psychosocial factors associated with pain intensity, pain-related interference, and psychological functioning in persons with multiple sclerosis and pain. Pain. 2007; 127(1):52-62. DOI: 10.1016/j.pain.2006.07.017 [PubMed: 16950570]

Pressman SD, Cohen S. Does positive affect influence health? Psychological Bulletin. 2005; 131(6): 925-971. DOI: 10.1037/0033-2909.131.6.925 [PubMed: 16351329]

Quale AJ, Schanke AK. Resilience in the face of coping with a severe physical injury: A study of trajectories of adjustment in a rehabilitation setting. Rehabilitation Psychology. 2010; 55(1): 12.doi: 10.1037/a0018415 [PubMed: 20175630]

Rietberg MB, Van Wegen EE, Kwakkel G. Measuring fatigue in patients with multiple sclerosis: Reproducibility, responsiveness and concurrent validity of three Dutch self-report questionnaires. Disability and Rehabilitation. 2010; 32(22):1870-1876. DOI: 10.3109/09638281003734458 [PubMed: 20345240]

Romano JM, Molton IR, Alschuler KN, Jensen MP, Schmaling KB, Buchwald DS. Reported pain and fatigue behaviors mediate the relationship between catastrophizing and perceptions of solicitousness in patients with chronic fatigue. Journal of Pain. 2016; 17(3):328-335. DOI: 10.1016/j.jpain.2015.10.020 [PubMed: 26646398]

Sheikh MA, Abelsen B, Olsen JA. Differential recall bias, intermediate confounding, and mediation analysis in life course epidemiology: An analytic framework with empirical example. Frontiers in Psychology. 2016; 7:1828.doi: 10.3389/fpsyg.2016.01828 [PubMed: 27933010] 
Sjonnesen K, Berzins S, Fiest KM, Bulloch AG, Metz LM, Thombs BD, Patten SB. Evaluation of the 9-item Patient Health Questionnaire (PHQ-9) as an assessment instrument for symptoms of depression in patients with multiple sclerosis. Postgraduate Medicine. 2012; 124(5):69-77. DOI: 10.3810/pgm.2012.09.2595 [PubMed: 23095427]

Smeets RJ, Vlaeyen JW, Kester AD, Knottnerus JA. Reduction of pain catastrophizing mediates the outcome of both physical and cognitive-behavioral treatment in chronic low back pain. Journal of Pain. 2006; 7(4):261-271. DOI: 10.1016/j.jpain.2005.10.011 [PubMed: 16618470]

Sturgeon JA, Zautra AJ. State and trait pain catastrophizing and emotional health in rheumatoid arthritis. Annals of Behavioral Medicine. 2013; 45(1):69-77. DOI: 10.1007/s12160-012-9408-Z [PubMed: 22915012]

Sturgeon JA, Zautra AJ, Arewasikporn A. A multilevel structural equation modeling analysis of vulnerabilities and resilience resources influencing affective adaptation to chronic pain. Pain. 2014; 155(2):292-298. DOI: 10.1016/j.pain.2013.10.007 [PubMed: 24120460]

Sullivan MJL. The Pain Catastrophizing Scale: User Manual. 1995

Sullivan MJ, Stanish W, Waite H, Sullivan M, Tripp DA. Catastrophizing, pain, and disability in patients with soft-tissue injuries. Pain. 1998; 77(3):253-260. DOI: 10.1016/ S0304-3959(98)00097-9 [PubMed: 9808350]

Tack B. A measure of fatigue in rheumatoid arthritis. Arthritis Care and Research. 1990; 3:S13.

Tan G, Jensen MP, Thornby JI, Shanti BF. Validation of the Brief Pain Inventory for chronic nonmalignant pain. Journal of Pain. 2004; 5(2):133-137. DOI: 10.1016/j.jpain.2003.12.005 [PubMed: 15042521]

Téllez N, Río J, Tintoré M, Nos C, Galán I, Montalban X. Does the Modified Fatigue Impact Scale offer a more comprehensive assessment of fatigue in MS. Multiple Sclerosis. 2005; 11(2):198202. DOI: 10.1191/1352458505ms1148oa [PubMed: 15794395]

Terrill AL, Molton IR, Ehde DM, Amtmann D, Bombardier CH, Smith AE, Jensen MP. Resilience, age, and perceived symptoms in persons with long-term physical disabilities. Journal of Health Psychology. 2014; 21(5):640-649. DOI: 10.1177/1359105314532973 [PubMed: 27165965]

Turk, DC. Cognitive-behavioral techniques in the management of pain. In: Foreyt, JP., Rathjen, DP., editors. Cognitive-Behavior Therapy: Research and Application. New York, NY: Plenum Press; 1978. p. 199-232.

Turner JA, Holtzman S, Mancl L. Mediators, moderators, and predictors of therapeutic change in cognitive-behavioral therapy for chronic pain. Pain. 2007; 127(3):276-286. DOI: 10.1016/j.pain. 2006.09.005 [PubMed: 17071000]

van den Akker LE, Beckerman H, Collette EH, Eijssen ICJM, Dekker J, de Groot V. Effectiveness of cognitive behavioral therapy for the treatment of fatigue in patients with multiple sclerosis: A systematic review and meta-analysis. Journal of Psychosomatic Research. 2016; 90:33-42. DOI: 10.1016/j.jpsychores.2016.09.002 [PubMed: 27772557]

Van Kessel K, Moss-Morris R. Understanding multiple sclerosis fatigue: A synthesis of biological and psychological factors. Journal of Psychosomatic Research. 2006; 61(5):583-585. DOI: 10.1016/ j.jpsychores.2006.03.006 [PubMed: 17084134]

Walsh MV, Armstrong TW, Poritz J, Elliott TR, Jackson WT, Ryan T. Resilience, pain interference, and upper limb loss: Testing the mediating effects of positive emotion and activity restriction on distress. Archives of Physical Medicine and Rehabilitation. 2016; 97(5):781-787. DOI: 10.1016/ j.apmr.2016.01.016 [PubMed: 26854856]

Watson D, Clark LA, Tellegen A. Development and validation of brief measures of positive and negative affect: The PANAS scales. Journal of Personality and Social Psychology. 1988; 54(6): 1063-1070. [PubMed: 3397865]

Williams RM, Turner AP, Hatzakis M, Bowen JD, Rodriquez AA, Haselkorn JK. Prevalence and correlates of depression among veterans with multiple sclerosis. Neurology. 2005; 64:75-80. [PubMed: 15642907]

Yeung EW, Arewasikporn A, Zautra AJ. Resilience and chronic pain. Journal of Social and Clinical Psychology. 2012; 31(6):593-617. DOI: 10.1521/jscp.2012.31.6.593 
Zautra AJ, Fasman R, Parish BP, Davis MC. Daily fatigue in women with osteoarthritis, rheumatoid arthritis, and fibromyalgia. Pain. 2007; 128(1):128-135. DOI: 10.1016/j.pain.2006.09.004 [PubMed: 17055648] 


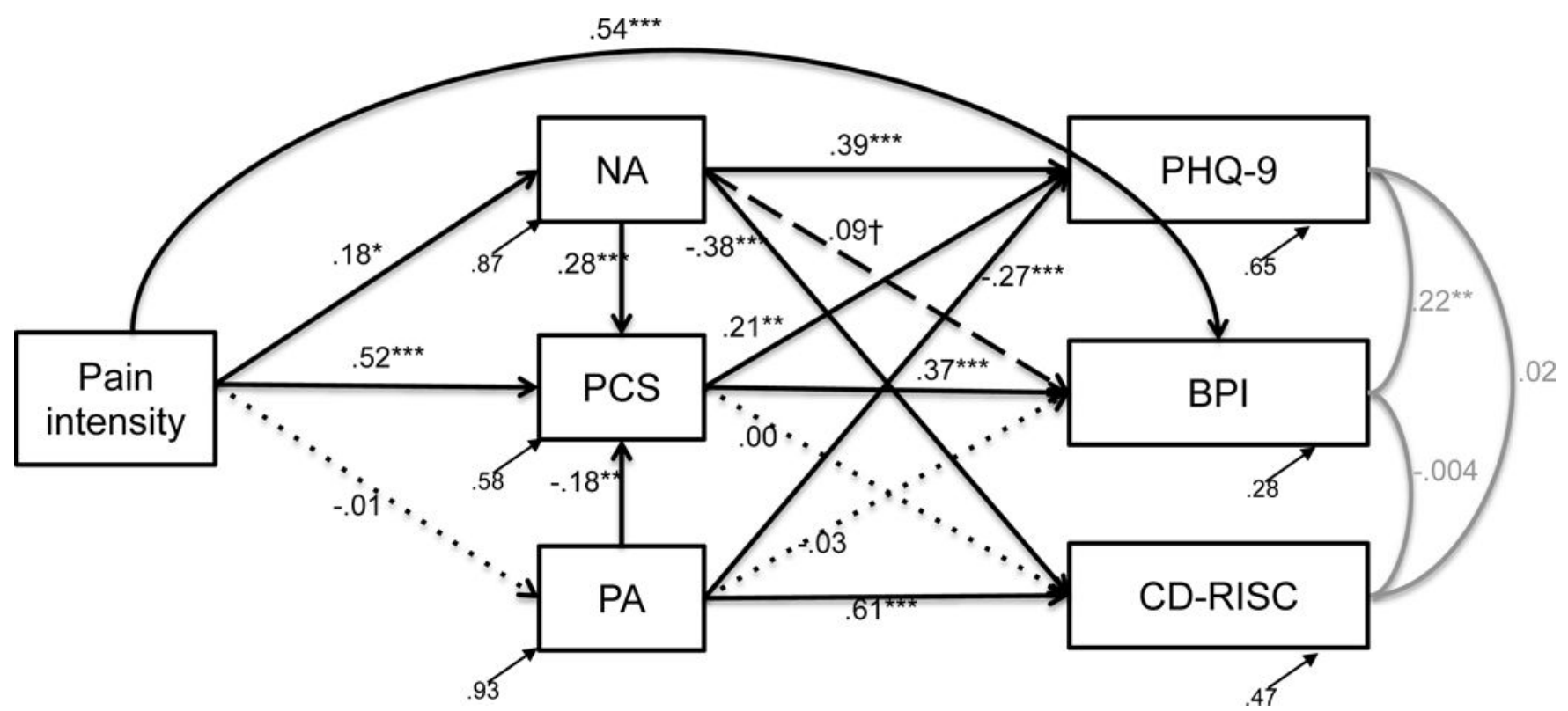

Figure 1.

Pain model with standardized parameter estimates and residuals, $\chi^{2}(31)=19.66, p=.018$, $\mathrm{RMSEA}=.061, \mathrm{CFI}=.965, \mathrm{SRMR}=.060$. Black solid lines denote significant pathways, black dashed lines denote marginally significant paths, and black dotted lines denote nonsignificant paths. Gray lines signify associations between the outcome variables. $\dagger p<.10$, $* p<.05, * * p<.01, * * * p<.001 ; \mathrm{PCS}=$ Pain Catastrophizing Scale; NA = negative affect; PA = positive affect; BPI = Body Pain Inventory pain interference; PHQ-9 = Patient Health Questionnaire-9 depression scale; CD-RISC = Connor Davidson Resilience scale 


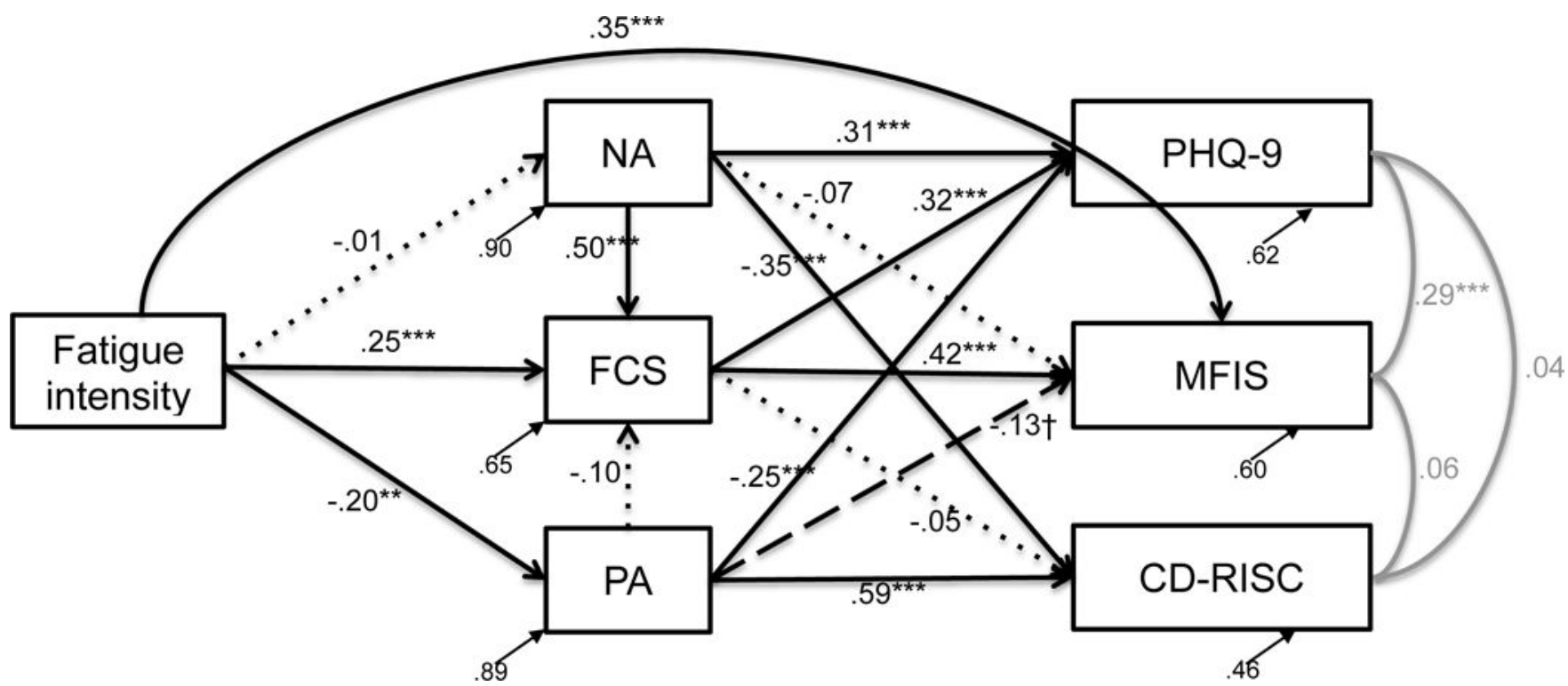

Figure 2.

Fatigue model with standardized parameter estimates and residuals, $\chi^{2}(27)=59.97, p=$. $0003, \mathrm{RMSEA}=.087, \mathrm{CFI}=.925, \mathrm{SRMR}=.066$. Black solid lines denote significant pathways, black dashed lines denote marginally significant paths, and black dotted lines denote nonsignificant paths. Gray lines signify associations between the outcome variables. $\dagger p<.10, * p<.05, * * p<.01, * * * p<.001 ; \mathrm{FCS}=$ fatigue catastrophizing; NA = negative affect; $\mathrm{PA}=$ positive affect ; MFIS = Modified Fatigue Impact Scale; PHQ-9 = Patient Health Questionnaire-9 depression scale; CD-RISC = Connor Davidson Resilience scale 


\section{Table 1}

Demographic and Disease-Related Characteristics of Sample $(n=163)$

\begin{tabular}{ll}
\hline Sample Characteristics & $\boldsymbol{n}(\boldsymbol{\%})$ \\
\hline Race & $136(83.4)$ \\
Non-Hispanic White & $19(11.7)$ \\
Non-Hispanic Black & $3(1.8)$ \\
Hispanic (1 or more race) & $5(3.1)$ \\
Non-Hispanic more than 1 race & \\
Education & $15(9.2)$ \\
High school or less & $42(25.8)$ \\
Tech or some college & $106(65.0)$ \\
College degree or higher & \\
Annual household income & \\
$<\$ 25,000$ & $32(19.6)$ \\
$2 \$ 25,000$ & $108(66.2)$ \\
Patient Determined Disease Steps ${ }^{a}$ & median: gait disability \\
No disability & $7(4.3)$ \\
Mild disability & $27(16.6)$ \\
Moderate disability & $21(12.9)$ \\
Gait disability & $48(29.4)$ \\
Early cane & $25(15.3)$ \\
Late cane & $14(8.6)$ \\
Bilateral support & $7(4.3)$ \\
Wheelchair/scooter & $13(8.0)$ \\
Note. & \\
Percentage may not add up to $100 \%$ & due to missing data. \\
&
\end{tabular}

Health Psychol. Author manuscript; available in PMC 2019 June 01. 